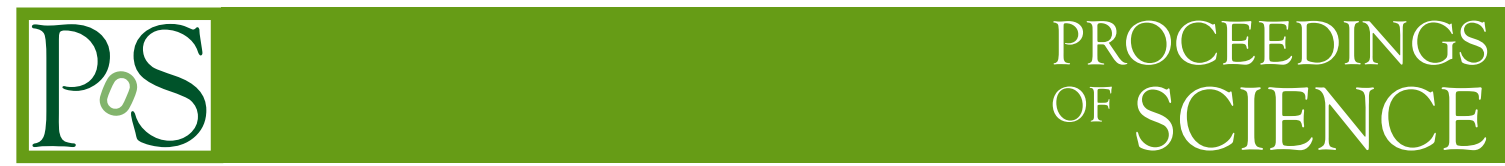

\title{
Charged particle identification (PID) for SuperB
}

\author{
Nicolas ARNAUD*i \\ Laboratoire de l'Accélérateur Linéaire, CNRS/IN2P3 et Université Paris-Sud - Orsay, France \\ E-mail: narnaudelal.in2p3.fr
}

\begin{abstract}
Charged particle identification (PID) is a key input for the physics program of the SuperB experiment. In the barrel region, the main detector for $\mathrm{K} / \pi$ separation will be the 'Focusing Detector of Internally Reflected Cherenkov light' (FDIRC), the successor of the successful BABAR DIRC, which camera has been completely re-designed. In addition, $R \& D$ is ongoing to add a detector on the forward side of SuperB to extend the PID coverage in this region. This innovative time-of-flight detector will use new electronics accurate at the 10-ps level.
\end{abstract}

XXIst International Europhysics Conference on High Energy Physics

21-27 July 2011

Grenoble, Rhône-Alpes France

\footnotetext{
*Speaker.

$\dagger$ On behalf of the SuperB PID collaboration.
} 


\section{Introduction}

The SuperB detector $[1,2]$ is being designed by an international collaboration which improves and optimizes the layout of the successful BABAR detector [3]. In spite of the more complex running conditions (a reduced boost, a luminosity two orders of magnitude higher and possibly larger backgrounds), its performances should be better than those of BABAR. The identification of charged particles (PID) is a key ingredient of the SuperB physics program [4, 5]. Various subdetectors can contribute to its performances: the tracking system (through the measurement of the energy lost by particles along their paths), the electromagnetic calorimeter (electrons) and the instrumented flux return (muons). In addition, a system dedicated to the $K / \pi$ separation up to $3-4 \mathrm{GeV} / \mathrm{c}$ covers the SuperB barrel region: the "Focusing Detector of Internally Reflected Cherenkov light" (FDIRC), the successor of the successful BABAR DIRC [6]. Finally, adding a new PID detector on the forward side of SuperB would improve the PID performances in a region where only $d E / d x$ measurements could be used otherwise. The candidate technology, based on time-of-flight measurements made possible by the use of ultra-fast and precise photon detectors and front-end electronics, has been selected by the SuperB collaboration in May 2011 and room has been reserved in the SuperB design to accomodate this new detector on the forward side. Tests of a full-scale prototype of one sector of the "Forward Time-Of-Flight" (FTOF) detector $\left(1 / 12^{\text {th }}\right.$ of the total) are foreseen for the coming months; if they are successful, the FTOF will be included in the SuperB baseline.

\section{The SuperB Focusing DIRC (FDIRC)}
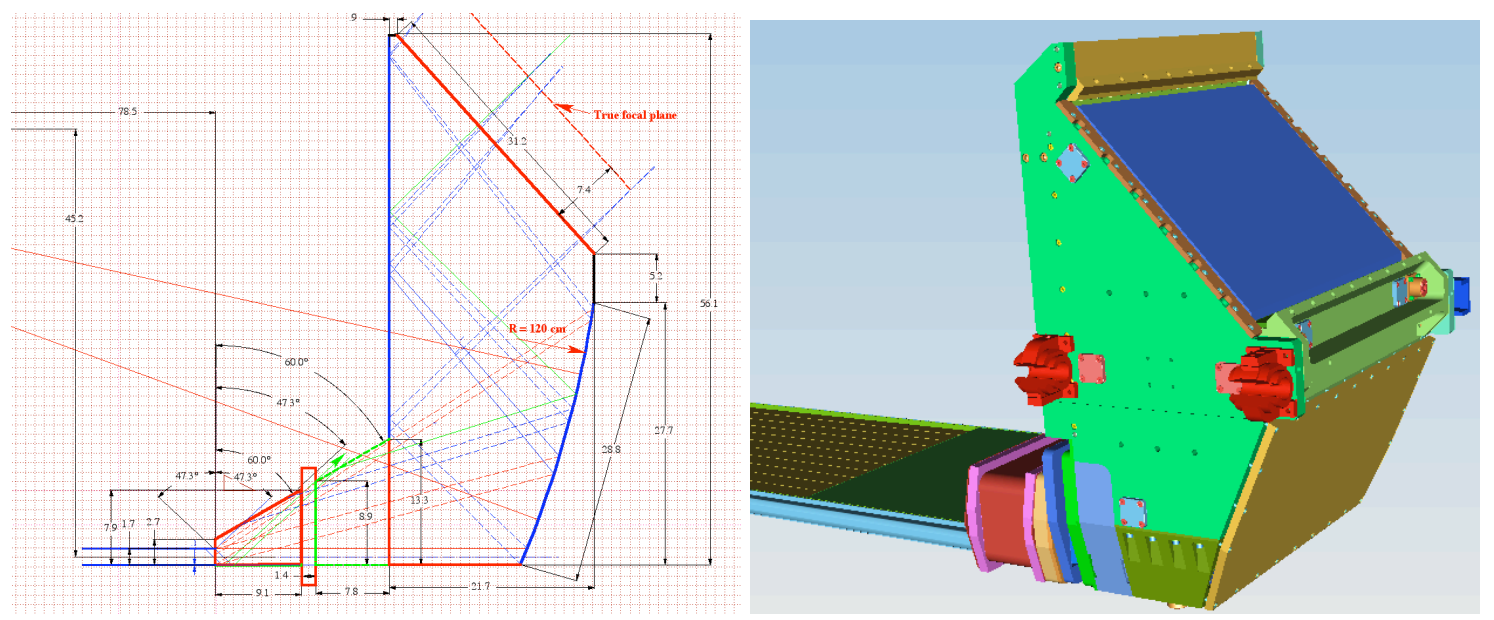

Figure 1: Left: design of the new FDIRC camera, a block of quartz with two mirrors (in blue; one cylindrical and the other plane) directing the Cherenkov photons to an array of photomultipliers (in red) close to the focal plane of the device. Right: engineering drawing of the FDIRC prototype (a full-size FBLOCK connected to a spare DIRC barbox) which will be tested in the SLAC Cosmic Ray Telescope in 2012.

A charged track crossing a DIRC quartz bar produces Cherenkov light inside. Part of the photons are trapped by total internal reflection and propagate until the bar exit window where they enter a large volume of ultra-pure water which inner surface is populated by about 11,000 
photomultipliers (PMTs). To cope with the higher luminosity and background (such as to get rid of all concerns about potential water leaks to the rest of the detector), the DIRC camera has been completely redesigned for SuperB: the water tank is replaced by 12 much smaller (the total volume is reduced by a factor of $\times 25$ ) quartz volumes, the FBLOCKs. Each FBLOCK will be glued to a DIRC barbox hosting 12 quartz bars and covering $30^{\circ}$ in azimuth of the barrel region. All barboxes have been removed from the BABAR detector during Fall 2010 and are now safely stored in a trailer which atmosphere is controlled - in particular a continuous nitrogen flow protects the quartz bars from humidity. The current design of the FBLOCK is based on ray-tracing [7], the experience gained with the operation of a first focusing DIRC prototype at SLAC [8] and a more recent Geant4-based simulation [9]. Each FBLOCK will be readout by 48 highly pixilated $\mathrm{H}$ 8500 MaPMTs from Hamamatsu. Thanks to the fast MaPMTs and the new front-end electronics (designed to cope with a $1 \mathrm{MHz}$ rate per channel), the photon arrival times will be known with an accuracy of $\approx 170 \mathrm{ps}$, to be compared with the $1.5 \mathrm{~ns}$ precision for the BABAR DIRC. The final resolution on the Cherenkov angle should be around 9 mrad per photon and 2.5-3 mrad per track. To validate the FBLOCK design, a full-scale prototype of one sector $\left(1 / 12^{\text {th }}\right.$ of the total $)$ is being assembled in the SLAC Cosmic Ray Telescope [10]; data taking should start in early 2012.

\section{Conclusion}

The PID is a key system for the SuperB detector. In the barrel region its main component is the FDIRC, based on the well-know DIRC design. After a complete redesign of its camera - the FDIRC reuses the original DIRC quartz bars - a full-scale prototype of one FDIRC sector will start taking data in early 2012 to validate this new detector concept and to allow the SuperB PID group to move from the $R \& D$ period to the construction phase. In addition, work is in progress to develop a new PID detector based on TOF measurements; if the foreseen prototype tests are successful, it will be added to the SuperB baseline layout.

\section{References}

[1] E. Grauges et al., The SuperB Detector Progress Report, arXiv: 1007.4241 (2010).

[2] N. Arnaud, "The SuperB Detector" in these proceedings.

[3] B. Aubert et al., (the BABAR Collaboration), Nuclear Instruments and Methods A 479 (2002) 1-116.

[4] B.O'Leary et al., The SuperB Physics Progress Report, arXiv:1008.1541 (2010).

[5] B. Meadows et al., The impact of SuperB on flavour physics, arXiv:1109. 5028 (2011).

[6] I. Adam et al., Nuclear Instruments and Methods A 538, 281 (2005).

[7] J. Va'vra, "Focusing DIRC design for SuperB", SLAC-PUB-13763 (2009).

[8] J. Benitez et al., "Development of a Focusing DIRC", SLAC-PUB-12236 (2006).

[9] J. Va'vra, D. Roberts and B. Ratcliff, "FDIRC design for SuperB", SLAC-PUB-14282 (2010).

[10] J. Va’vra, "SLAC Cosmic Ray Telescope Facility", SLAC-PUB-13873 (2010). 